\title{
Asymptotic Inverse Problem for Almost-Periodically Perturbed Quantum Harmonic Oscillator
}

\author{
Alexis Pokrovski *
}

\begin{abstract}
Let $\left\{\mu_{n}\right\}_{n=0}^{\infty}$ be the spectrum of $-\frac{d^{2}}{d x^{2}}+x^{2}+q(x)$ in $L^{2}(\mathbb{R})$, where $q$ is an even almostperiodic complex-valued function with bounded primitive and derivative. Suppose that the asymptotic approximation to the first asymptotic correction $\Delta \mu_{n}=\mu_{n}-\mu_{n}^{0}+$ $o\left(n^{-\frac{1}{4}}\right), \mu_{n}^{0}=2 n+1$ is known. We prove the formula that gives the frequencies and the Fourier coefficients of $q$ in terms of $\Delta \mu_{n}$.
\end{abstract}

AMS Mathematics Subject Classifications (1991): 34L20 (Asymptotic distribution of eigenvalues, asymptotic theory of eigenfunctions);

81Q15 (Perturbation theories for operators and differential equations).

Key words: almost-periodic perturbation; inverse problem; quantum harmonic oscillator; spectral asymptotics.

\section{Introduction and main result}

Consider the operator describing perturbed quantum harmonic oscillator

$$
A=-\frac{d^{2}}{d x^{2}}+x^{2}+q(x) \quad \text { in } \quad L^{2}(\mathbb{R})
$$

with the perturbation $q(x)$ from the class $\mathcal{B}=\left\{q:\left\|q^{\prime}\right\|_{\infty}+\|Q\|_{\infty}<\infty\right\}$, where $Q(x)=\int_{0}^{x} q d t$ and $\|\cdot\|_{\infty}$ denotes the norm in $L^{\infty}(\mathbb{R})$. It was proved in [1] that the spectrum $\left\{\mu_{n}\right\}_{n=0}^{\infty}$ of $A$ has the asymptotics $\mu_{n}=\mu_{n}^{0}+\mu_{n}^{1}+O\left(n^{-\frac{1}{3}}\right)$, where $\mu_{n}^{0}=2 n+1$ and $\mu_{n}^{1}=O\left(n^{-\frac{1}{4}}\right)$.

For the perturbations that are sum of almost-periodic and decaying terms we study the problem of recovering of the almost-periodic part from the first asymptotic correction $\mu_{n}^{1}$. Specifically, we consider the perturbations

$$
q=p+r \in \mathcal{B}, \quad p \in B^{1}, p(-x)=p(x) \quad \text { and } \quad\|r\|_{B^{1}} \equiv \lim _{T \rightarrow \infty} \frac{1}{2 T} \int_{-T}^{T}|r(x)| d x=0,
$$

*Laboratory of Quantum Networks, Institute for Physics, St-Petersburg State University, St.Petersburg 198504, Ulyanovskaya 1. E-mail: pokrovsk@AP15398.spb.edu 
where $B^{1}$ is the Besikovitch space of almost-periodic functions [2] (closure of trigonometric polynomials $\sum_{k=0}^{N} a_{k} e^{i \nu_{k} x}, \nu_{k}$ real, in the norm $\left.\|\cdot\|_{B^{1}}\right)$. It is sufficient to recover $p$ in terms of its Fourier transform [2]. Here is the main result.

Theorem 1.1. Let $\left\{\Delta \mu_{n}\right\}_{n=N}^{\infty}$ approximates the first asymptotic correction to the spectrum of the operator (1.1), (1.2):

$$
\Delta \mu_{n}=\mu_{n}-\mu_{n}^{0}+o\left(n^{-\frac{1}{4}}\right) .
$$

Then the spectrum and the Fourier coefficients of the almost-periodic part $p$ can be recovered from the relation

$$
\lim _{L \rightarrow \infty} \frac{1}{x_{L}} \sum_{n=N}^{L-1} \Delta \mu_{n} G_{\nu}\left(x_{n}, x_{L}\right)\left(x_{n+1}-x_{n}\right)=\lim _{T \rightarrow \infty} \frac{1}{T} \int_{0}^{T} p(t) \cos \nu t d t, \quad \nu \geqslant 0,
$$

where $x_{n}=\sqrt{\mu_{n}^{0}}=\sqrt{2 n+1}, G_{\nu}(x, T)=-x \int_{x}^{T} \frac{\varphi_{\nu, T}^{\prime}(t) d t}{\sqrt{t^{2}-x^{2}}}, \varphi_{\nu, T}(t)=\eta(t-T) \cos \nu t$ and $\eta \in C^{2}(\mathbb{R})$ is a smoothed step function such that $\eta(t)=1$ for $x \in(-\infty,-1], \eta(t)=0$ for $x \in[0, \infty)$ and $\eta^{\prime}(0)=0$.

Asymptotic inverse spectral problem for quantum harmonic oscillator with slowly decaying perturbation was considered by Gurarie [3. He studied the operator (1.1) with real $q(x) \sim|x|^{-\alpha} \sum a_{m} \cos \omega_{m} x$ for $|x| \rightarrow \infty$, where $\alpha>0$ and the sum in the numerator is finite. The approach in [3] is based on the spectral asymptotics

$$
\mu_{n}=\frac{\widetilde{q}(\sqrt{2 n})}{n^{1 / 4+\alpha / 2}}+O\left(\frac{1}{\sqrt{n}}\right), \quad \widetilde{q}(x)=\mathrm{const} \sum \frac{a_{m}}{\sqrt{\omega_{m}}} \cos \left(\omega_{m} x-\pi / 4\right)
$$

which exhibits linear relation between the leading asymptotic terms of $q$ and $\mu_{n}$. However, the technique of [3] does not cover the case $\alpha=0$.

We consider just this case in a slightly more general setting (almost-periodic functions vs. finite trigonometric sums). Our method also allows complex-valued $q$. Technically, the result is based on the recent proof [1] of the spectral asymptotics

$$
\mu_{n}=\mu_{n}^{0}+\frac{1}{2 \pi} \int_{-\pi}^{\pi} q\left(\sqrt{\mu_{n}^{0}} \sin \vartheta\right) d \vartheta+O\left(n^{-\frac{1}{3}}\right), \quad \text { for } \quad q \in \mathcal{B}
$$

Thus the proof of Theorem 1.1 follows from the asymptotic behavior of the integral in (1.5), which is analyzed in Lemmas 2.1 and 2.3 .

\section{Properties of the Schlömilch integral}

The integral in the spectral asymptotics (1.5) is the Schlömilch integral [4]

$$
g_{q}(x)=\frac{2}{\pi} \int_{0}^{\pi / 2} q_{+}(x \sin \vartheta) d \vartheta=\frac{2}{\pi} \int_{0}^{x} \frac{q_{+}(t) d t}{\sqrt{x^{2}-t^{2}}}, \quad q_{+}(x)=(q(x)+q(-x)) / 2
$$


evaluated at the points $x_{n}=\sqrt{2 n+1}$. In the next Lemma we estimate the integral and its derivatives. Then in Lemma 2.3 we prove similar estimates for the inverse Schlömilch integral. (We could not find in the literature the results of these Lemmas for the specific class $\mathcal{B}$.) Using the two Lemmas, we prove Theorem 1.1.

Everywhere below $C$ denotes an absolute constant.

Lemma 2.1. Let $f \in \mathcal{B}$ and $g(x)=\int_{0}^{\pi / 2} f(x \sin \vartheta) d \vartheta$. Then

$$
|g(x)| \leqslant C \frac{\|F\|_{\infty}+\|f\|_{\infty}}{\sqrt{1+x}}, \quad\left|g^{\prime}(x)\right| \leqslant C \frac{\|f\|_{\infty}+\left\|f^{\prime}\right\|_{\infty}}{\sqrt{1+x}}, \quad x>0,
$$

where $F(x)=\int_{0}^{x} f d t$.

Proof. For $x \leqslant 1$ the result is evident, so we consider only the case $x>1$. Using the change of variables $t=\sin \vartheta$, we write $g(x)=\int_{0}^{1} \frac{f(x t) d t}{\sqrt{1-t^{2}}}$ and split it as

$$
g=I_{1}+I_{2}, \quad I_{1}=\int_{0}^{1-\varepsilon} \frac{f(x t) d t}{\sqrt{1-t^{2}}}, \quad I_{2}=\int_{1-\varepsilon}^{1} \frac{f(x t) d t}{\sqrt{1-t^{2}}}
$$

where $\varepsilon=1 / x$. Using the notation $(\ldots)_{t}^{\prime}$ for $\frac{\partial}{\partial t}(\ldots)$ and choosing the primitive $\widetilde{F}$ of $f$, satisfying $\widetilde{F}(x(1-\varepsilon))=0$, we have

$$
\begin{array}{r}
I_{1}=\frac{1}{x} \int_{0}^{1-\varepsilon}(\widetilde{F}(x t))_{t}^{\prime} \frac{d t}{\sqrt{1-t^{2}}}=\frac{1}{x}\left(\left.\frac{\widetilde{F}(x t)}{\sqrt{1-t^{2}}}\right|_{t=0} ^{t=1-\varepsilon}+\int_{0}^{1-\varepsilon} \frac{\widetilde{F}(x t) t d t}{\left(1-t^{2}\right)^{3 / 2}}\right) . \text { Therefore, } \\
\left|I_{1}\right| \leqslant 2 \frac{\|F\|_{\infty}}{x}\left(1+\int_{\varepsilon}^{1} \frac{d t}{t^{3 / 2}}\right) \leqslant C \frac{\|F\|_{\infty}}{x}\left(1+\frac{1}{\sqrt{\varepsilon}}\right) .
\end{array}
$$

Now we substitute the estimate $\left|I_{2}\right| \leqslant\|f\|_{\infty} \int_{0}^{\varepsilon} \frac{d t}{\sqrt{t}}=2\|f\|_{\infty} / \sqrt{x}$ and (2.9) into (2.8). This gives the first inequality in (2.7). We prove the second one in a similar way, writing

$$
g^{\prime}=I_{1}^{\prime}+I_{2}^{\prime}, \quad I_{1}^{\prime}=\int_{0}^{1-\varepsilon} \frac{t f^{\prime}(x t) d t}{\sqrt{1-t^{2}}}, \quad I_{2}^{\prime}=\int_{1-\varepsilon}^{1} \frac{t f^{\prime}(x t) d t}{\sqrt{1-t^{2}}}, \quad \varepsilon=1 / x .
$$

We integrate by parts in $I_{1}^{\prime}$, choosing the primitive $\widetilde{f}(x t)=f(x t)-f(x(1-\varepsilon))$. This gives $I_{1}^{\prime}=-\frac{1}{x} \int_{0}^{1-\varepsilon} \frac{\widetilde{f}(x t) d t}{\left(1-t^{2}\right)^{3 / 2}}$, hence

$$
\left|I_{1}^{\prime}\right| \leqslant C \frac{\|f\|_{\infty}}{x}\left(1+\frac{1}{\sqrt{\varepsilon}}\right) .
$$

We substitute the estimate $\left|I_{2}^{\prime}\right| \leqslant C\left\|f^{\prime}\right\|_{\infty} / \sqrt{x}$ and (2.11) in (2.10). This gives the second inequality in (2.7).

Remark 2.2. The rate of decay $x^{-1 / 2}$ as $x \rightarrow \infty$ in (2.7) cannot be improved. (The example $f(x)=\cos x$ gives the Bessel function $J_{0}$.) 
Lemma 2.3. Let $T>2, \varphi, \varphi^{\prime \prime} \in L^{\infty}([0, T])$ and $\varphi(T)=0$. Then the equation $\varphi(t)=$ $\frac{2}{\pi} \int_{t}^{T} \frac{g(x) d x}{\sqrt{x^{2}-t^{2}}}$ has the unique solution $g(x)=-x \int_{x}^{T} \frac{\varphi^{\prime}(t) d t}{\sqrt{t^{2}-x^{2}}}$ for $x \in[0, T]$, such that

$$
|g(x)| \leqslant C\left(\|\varphi\|_{\infty}+\left\|\varphi^{\prime}\right\|_{\infty}\right) \sqrt{x}, \quad \text { for } \quad x>1 .
$$

If, in addition, $\varphi^{\prime}(T)=0$, then

$$
\left|g^{\prime}(x)\right| \leqslant C\left(\left\|\varphi^{\prime}\right\|_{\infty}+\left\|\varphi^{\prime \prime}\right\|_{\infty}\right) \sqrt{x}, \quad \text { for } \quad x>1 .
$$

Proof. In terms of $\widetilde{g}(x)=\frac{g(\sqrt{x})}{2 \sqrt{x}}$ and $\widetilde{\varphi}(t)=\varphi(\sqrt{t})$ the equation on $g$ becomes the Abel equation $\widetilde{\varphi}(t)=\frac{2}{\pi} \int_{t}^{T^{2}} \frac{\widetilde{g}(s) d s}{\sqrt{s-t}}$. Its solution for absolutely continuous $\widetilde{\varphi}$ is $\widetilde{g}(s)=\frac{\widetilde{\varphi}\left(T^{2}\right)}{\sqrt{T^{2}-s}}-$ $\int_{s}^{T^{2}} \frac{\widetilde{\varphi}^{\prime}(u) d u}{\sqrt{u-s}}$ (see Ch.1, $\S 2$ of [5]). Using $\varphi(T)=0$, we obtain the required formula for $g$.

Consider (2.12). For $x \in[T-1, T]$ the inequality follows from the direct estimate $\left|\frac{g(x)}{x}\right| \leqslant \frac{\left\|\varphi^{\prime}\right\|_{\infty}}{\sqrt{2 x}} \int_{T-1}^{T} \frac{d t}{\sqrt{t-(T-1)}}$. For $x \in[0, T-1]$ write

$$
-\frac{g(x)}{x}=I_{1}+I_{2}, \quad I_{1}=\int_{x}^{x+1} \frac{\varphi^{\prime}(t) d t}{\sqrt{t^{2}-x^{2}}}, \quad I_{2}=\int_{x+1}^{T} \frac{\varphi^{\prime}(t) d t}{\sqrt{t^{2}-x^{2}}}
$$

and integrate $I_{2}$ by parts. We have $I_{2}=\left.\frac{\varphi(t)}{\sqrt{t^{2}-x^{2}}}\right|_{t=x+1} ^{t=T}-\int_{x+1}^{T} \varphi(t) \frac{\partial}{\partial t} \frac{1}{\sqrt{t^{2}-x^{2}}} d t$. Therefore,

$$
\left|I_{2}\right| \leqslant \frac{\|\varphi\|_{\infty}}{\sqrt{1+2 x}}+\left.\|\varphi\|_{\infty} \frac{(-1)}{\sqrt{t^{2}-x^{2}}}\right|_{t=x+1} ^{t=\infty} \leqslant \frac{2\|\varphi\|_{\infty}}{\sqrt{1+2 x}}
$$

Now we substitute the estimate $\left|I_{1}\right| \leqslant\left\|\varphi^{\prime}\right\|_{\infty} \int_{x}^{x+1} \frac{d t}{\sqrt{t^{2}-x^{2}}} \leqslant\left\|\varphi^{\prime}\right\|_{\infty} \sqrt{2 / x}$ and (2.15) into (2.14). This gives (2.12), as required.

Next consider (2.13). By $g^{\prime}(x)=x(g(x) / x)^{\prime}+g(x) / x$ and (2.12), it is sufficient to estimate $x(g(x) / x)^{\prime}$. Using $\varphi^{\prime}(T)=0$, we obtain

$$
x(g(x) / x)^{\prime}=-\frac{T \varphi^{\prime}(T)}{\sqrt{T^{2}-x^{2}}}+\int_{1}^{T / x} \frac{x s \varphi^{\prime \prime}(x s) d s}{\sqrt{s^{2}-1}}=\int_{x}^{T} \frac{t \varphi^{\prime \prime}(t) d t}{\sqrt{t^{2}-x^{2}}} .
$$

For $x \in[T-1, T]$ we have $\left|x(g(x) / x)^{\prime}\right| \leqslant \frac{2 x}{\sqrt{2 x}} \int_{T-1}^{T} \frac{\left|\varphi^{\prime \prime}(t)\right| d t}{\sqrt{t-(T-1)}} \leqslant 2 \sqrt{2}\left\|\varphi^{\prime \prime}\right\|_{\infty} \sqrt{x}$. For $x \in$ $[0, T-1]$ write

$$
x(g(x) / x)^{\prime}=I_{1}^{\prime}+I_{2}^{\prime}, \quad I_{1}^{\prime}=\int_{x}^{x+1} \frac{t \varphi^{\prime \prime}(t) d t}{\sqrt{t^{2}-x^{2}}}, \quad I_{2}^{\prime}=\int_{x+1}^{T} \frac{t \varphi^{\prime \prime}(t) d t}{\sqrt{t^{2}-x^{2}}}
$$

and take the integral for $I_{2}^{\prime}$ by parts. We have $I_{2}^{\prime}=-\frac{(x+1) \varphi^{\prime}(x+1)}{\sqrt{(x+1)^{2}-x^{2}}}+\int_{x+1}^{T} \varphi^{\prime}(t) \frac{\partial}{\partial t} \frac{t}{\sqrt{t^{2}-x^{2}}} d t$. Hence, using $\frac{\partial}{\partial t} \frac{t}{\sqrt{t^{2}-x^{2}}} \leqslant 0$ we obtain

$$
\left|I_{2}^{\prime}\right| \leqslant \frac{(1+x)\left\|\varphi^{\prime}\right\|_{\infty}}{\sqrt{1+2 x}}+\left\|\varphi^{\prime}\right\|_{\infty} \int_{x+1}^{\infty} \frac{\partial}{\partial t} \frac{(-t)}{\sqrt{t^{2}-x^{2}}} d t \leqslant 2\left\|\varphi^{\prime}\right\|_{\infty} \sqrt{1+x} .
$$

Now we substitute the estimate $\left|I_{1}^{\prime}\right| \leqslant\left\|\varphi^{\prime \prime}\right\|_{\infty} \int_{x}^{x+1} \frac{t d t}{\sqrt{t^{2}-x^{2}}} \leqslant\left\|\varphi^{\prime \prime}\right\|_{\infty} \sqrt{1+2 x}$ and (2.18) into (2.17). This gives (2.13). 
Remark 2.4. Note that the condition $\varphi(T)=0$ is necessary for (2.12). If $\varphi(T) \neq 0$, then $g(x)$ is unbounded due to the non-integral term in the inversion formula for the Abel equation. The term is $O\left(\frac{\varphi(T)}{\sqrt{T-x}}\right)$ for $x \uparrow T$. Similarly, the condition $\varphi^{\prime}(T)=0$ is necessary for (2.13). If $\varphi^{\prime}(T) \neq 0$, then $g^{\prime}(x)$ is unbounded as $x \uparrow T$ due to the non-integral term $\frac{T \varphi^{\prime}(T)}{\sqrt{T^{2}-x^{2}}}$ in (2.16).

Proof of Theorem 1.1. Compare (1.3) with the asymptotis (1.5). It is clear that

$$
\Delta \mu_{n}=g_{q}\left(x_{n}\right)+o\left(n^{-\frac{1}{4}}\right), \quad x_{n}=\sqrt{\mu_{n}^{0}}=\sqrt{2 n+1},
$$

where the Schlömilch integral $g_{q}$ is given by (2.6). The proof is based on the fact that the set $\left\{x_{n}\right\}_{n=0}^{\infty}$ becomes arbitrarily dense as $n \rightarrow \infty$, so that Riemann sums

$\frac{1}{T} \sum_{x_{n+1} \leqslant T} G_{\nu}\left(x_{n}, T\right) g_{q}\left(x_{n}\right)\left(x_{n+1}-x_{n}\right)$ approximate $\frac{1}{T} \int_{0}^{T} G_{\nu}(x, T) g_{q}(x) d x$, provided the integrand is smooth enough. Using the inversion formula for the Schlömilch integral, we choose $G_{\nu}$ such that the last expression tends to $\frac{1}{T} \int_{0}^{T} q_{+}(t) \cos \nu t d t$ as $T \rightarrow \infty$.

By Lemma 2.3, we have

$$
\int_{0}^{T} G_{\nu}(x, T) g_{q}(x) d x=\int_{0}^{T} q_{+}(t)\left(\frac{2}{\pi} \int_{t}^{T} \frac{G_{\nu}(x, T) d x}{\sqrt{x^{2}-t^{2}}}\right) d t=\int_{0}^{T} q_{+}(t) \varphi_{\nu, T}(t) d t .
$$

Let us show that the integrand in the left-hand side of (2.20) is sufficiently smooth. By Lemma 2.1.

$$
\left|g_{q}(x)\right| \leqslant C \frac{\|Q\|_{\infty}+\|q\|_{\infty}}{\sqrt{1+x}}, \quad\left|g_{q}^{\prime}(x)\right| \leqslant C \frac{\|q\|_{\infty}+\left\|q^{\prime}\right\|_{\infty}}{\sqrt{1+x}}, \quad x \geqslant 0,
$$

where $C$ is an absolute constant. Similarly, since $\varphi_{\nu, T}$ satisfies the hypothesis of Lemma 2.3, uniformly in $T \geqslant x$

$$
\left|G_{\nu}(x, T)\right| \leqslant C(1+\nu) \sqrt{x}, \quad\left|\frac{\partial}{\partial x} G_{\nu}(x, T)\right| \leqslant C(1+\nu)^{2} \sqrt{x}, \quad x \geqslant 1,
$$

where we used $\left\|\varphi_{\nu, T}\right\|_{\infty} \leqslant C,\left\|\varphi_{\nu, T}^{\prime \prime}\right\|_{\infty} \leqslant C(1+\nu)^{2}$. Therefore, for any fixed $\nu$ the function $h_{T}(x) \stackrel{\text { def }}{=} G_{\nu}(x, T) g_{q}(x)$ and its $x$-derivative are uniformly bounded for $x \geqslant 1$ and $T \geqslant x$. Hence, for $T \rightarrow \infty$ we have

$$
\frac{1}{T} \sum_{x_{n+1} \leqslant T} h_{T}\left(x_{n}\right)\left(x_{n+1}-x_{n}\right)-\frac{1}{T} \int_{0}^{T} h_{T}(x) d x=\frac{1}{T} \int_{0}^{T} O\left(\frac{1}{x}\right) d x=O\left(\frac{\ln T}{T}\right) \rightarrow 0,
$$

where we used $x_{n+1}-x_{n}=O\left(x_{n}^{-1}\right)$. Now, by (2.23), (2.19) and the first estimate in (2.22),

$$
\lim _{T \rightarrow \infty} \frac{1}{T} \sum_{x_{n+1} \leqslant T} \Delta \mu_{n} G_{\nu}\left(x_{n}, T\right)\left(x_{n+1}-x_{n}\right)=\lim _{T \rightarrow \infty} \frac{1}{T} \int_{0}^{T} G_{\nu}(x, T) g_{q}(x) d x .
$$

Next we divide (2.20) by $T$ and take the limit $T \rightarrow \infty$. By (2.24) and $\lim _{T \rightarrow \infty} \frac{1}{T} \int_{0}^{T} q_{+} \varphi_{\nu, T} d t=$ $\lim _{T \rightarrow \infty} \frac{1}{T} \int_{0}^{T} p(t) \cos \nu t d t$, this gives (1.4)

Remark 2.5. We have $(\nu x)^{-1} G_{\nu}(x, \infty)=\frac{2}{\pi} \int_{x}^{\infty} \frac{\sin \nu t d t}{\sqrt{t^{2}-x^{2}}}=J_{0}(\nu x)$, where $J_{0}$ is the Bessel function (see e.g. [6]). 


\section{Acknowledgements}

The author is thankful to E.Korotyaev and S.Naboko for fruitful discussions and valuable advice.

\section{References}

[1] Klein, M., Korotyaev, E. and Pokrovski, A.: Spectral Asymptotics of the Harmonic Oscillator Perturbed by Bounded Potentials, Annales Henri Poincare, 6 (2005), 747 789. (arxiv.org/math-ph/0312066)

[2] Besikovitch, A.S.: Almost Periodic Functions, Dover Publ Inc., 1954.

[3] Gurarie, D.: Asymptotic Inverse Spectral Problem for Anharmonic Oscillators, Comm.Math.Phys., 112 (1987), 491-502.

[4] Whittaker, E.T. and Watson, G.N.: A Course of Modern Analysis Vol.1, Cambridge University Press, 1927.

[5] Samko S.G., Kilbas A.A., Marichev O.I.: Integrals and derivatives of fractional order and their applications (in Russian), Nauka i Tehnika, Minsk, 1987.

[6] Gradstein, I.S. and Ryzhik, I.M.: Tables of integrals, sums, series and products (in Russian), GIFML, Moscow, 1963. 